\title{
Transcriptomic analysis reveals moderate modulation of macrophage migration inhibitory factor superfamily genes in alcohol use disorders
}

\author{
MARIA CRISTINA PETRALIA ${ }^{1}$, EMANUELA MAZZON ${ }^{2}$, KATIA MANGANO ${ }^{2}$, \\ PAOLO FAGONE ${ }^{1}$, ROBERTO DI MARCO $^{3}$, LUCA FALZONE ${ }^{1}$, MARIA SOFIA BASILE ${ }^{1}$, \\ FERDINANDO NICOLETTI $^{1}$ and EUGENIO CAVALLI ${ }^{2}$
}

\author{
${ }^{1}$ Department of Biomedical and Biotechnological Sciences, University of Catania, I-95123 Catania; \\ ${ }^{2}$ IRCCS (Scientific Institute for Research, Hospitalization and Healthcare) Centro Neurolesi ‘Bonino-Pulejo', I-98124 Messina; \\ ${ }^{3}$ Department of Medicine and Health Sciences 'Vincenzo Tiberio', University of Molise, I-86100 Campobasso, Italy
}

Received September 12, 2019; Accepted November 14, 2019

DOI: $10.3892 /$ etm.2020.8410

\begin{abstract}
Alcohol use disorder (AUD) is a primary, chronic and relapsing disease of brain reward, motivation and memory, which is associated with several comorbidities, including major depression and post-traumatic stress disorder. It has been revealed that Ibudilast (IBUD), a dual inhibitor of phosphodiesterase-4 and -10 and of macrophage migration inhibitory factor (MIF), exerts beneficial effects on AUD in rodent models and human patients. Therefore, IBUD has attracted increasing interest, with research focusing on the elucidation of the pathogenic role of MIF and its homologue, D-dopachrome tautomerase (DDT), in the pathogenesis and maintenance of AUD. By using DNA microarray analysis, the current study performed a transcriptomic expression analysis of MIF, DDT and their co-receptors, including CD74, C-X-C chemokine receptor (CXCR)2, CXCR4 and CXCR7 in patients with AUD. The results revealed that the transcriptomic levels of MIF, DDT and their receptors were superimposable in the prefrontal cortex of rodents and patients with AUD and human patients. Furthermore, peripheral blood cells from heavy drinkers exhibited a moderate increase in MIF and DDT levels, both at the baseline and following exposure to alcohol-associated cues, based on individual situations that included alcohol-related stimuli resulting in subsequent alcohol use (buying alcohol and being at a bar, watching others drink alcohol). Considering the overlapping effects of MIF and DDT, the inverse Fisher's $\chi^{2}$ test was performed on
\end{abstract}

Correspondence to: Professor Ferdinando Nicoletti, Department of Biomedical and Biotechnological Sciences, University of Catania, 89 Via Santa Sofia, I-95123 Catania, Italy

E-mail: ferdinic@unict.it

Key words: alcohol use disorder, DNA microarray D-dopachrome tautomerase, immune modulation, macrophage migration inhibitory factor, psychotherapy unadjusted P-values to evaluate the combined effect of MIF and DDT. The results revealed a significant increase in these cytokines in heavy drinkers compared with controls (moderate drinkers). To the best of our knowledge, the present study demonstrated for the first time that MIF and DDT expression was upregulated in the blood of patients with AUD. These results therefore warrant further study to evaluate the role of MIF and DDT in the development and maintenance of AUD, to evaluate their use as biomarkers to predict the psychotherapeutic and pharmacological response of patients with AUD and for use as therapeutic targets.

\section{Introduction}

Alcohol-use disorder (AUD) is a major health problem worldwide characterized by a chronic relapsing course of the disease and it is associated to multiple abnormalities of brain reward, motivation and memory, along with the inability of AUD patients to regulate drinking, in spite of multiple negative consequences of alcohol abuse. AUD is accompanied by significant comorbidities and mortality, including depression and fetal alcohol spectrum disorders (FASD) (1). FASD entails multiple disabilities, including craniofacial and skeletal abnormalities and it represents the main cause of mental retardation in USA (1). In addition, approximately $50 \%$ of AUD patients also suffer of post-traumatic stress disorder (PTSD) $(2,3)$. The patients suffering from AUD and PTSD manifest greater severity of psychiatric pathologies and are more resistant to therapeutic interventions (4). AUD therefore represents a major health problem worldwide entailing social, personal and legal aspects.

The standard of care treatment for AUD consists of abstinence from alcohol and psychosocial approaches. Adjuvant pharmacological therapy is also available and recommended (5). The only drugs that are specifically approved for AUD are acamprosate, naltrexone and nalmefene, while disulfiram is considered as second line pharmacological approach. Several drugs are also being considered in clinical development for AUD patients, including topiramate, 
gabapentin, diazepam, ifenprodil, memantine, prazosine and $\mathrm{N}$-acetylcysteine, among others $(6,7)$. Nonetheless, the efficacy of medications for alcohol-dependence remains modest, and there are no reliable laboratoristic and/or clinical predictors of treatment response (5). It is expected that the limited pharmacological efficacy could be augmented through the better understanding of the pathophysiology of alcohol dependence, as well as through the identification of biomarkers of response to therapeutic treatments and by modalities that improve medication adherence (8). Along this line of research, studies are warranted that aim at identifying pathogenic and biomolecular mechanisms of alcohol dependence and that may help design novel tailored approaches for the treatment of this condition that could be used alone or in combination with existing treatment and psychotherapeutic approaches.

Inflammation and altered innate immune responses are hallmarks of alcohol-induced organ damage, affecting the liver, cardiovascular system, and brain, and immune abnormalities induced by alcohol exposure have been well documented both in rodent models and humans with AUD (9). In rodent models, it has been shown that increased systemic and brain production of proinflammatory cytokines such as TNF $\alpha$, IL-1 $\beta$ and MCP-1 and neuroinflammation occurs when 10-days ethanol administration is followed by endotoxin challenge. Alcohol exposure significantly augments the production of proinflammatory cytokines induced by LPS, with a long-lasting persistence in the brain. Furthermore, the brains of mice exposed to 10-days alcohol administration exhibited signs of microglia activation (10). In agreement with these data, it has been shown that in vivo administration of alcohol to adult male Wistar rats $(1.0 \mathrm{~g} / \mathrm{kg})$ once daily for 14 days increased the concentrations of the proinflammatory cytokines (IL- $1 \beta$ and TNF- $\alpha$ ) in two primary brain regions, such as the hippocampus and frontal cortex, that are implicated in mood regulation (11). In humans, an increase in serum proinflammatory cytokines including TNF $\alpha$, IL-1 $\beta$ occurs upon prolonged alcohol exposure (12) and the monocytes isolated from the blood of alcoholics produce greater amounts of $\mathrm{TNF} \alpha$, both spontaneously and in response to endotoxin challenge (13). Chronic alcohol consumption elevates the levels of circulating TNF $\alpha$, IL-1 $\beta$ and IL- 6 both in the woman and in their fetuses (14). It is also known that AUD patients exhibit abnormal profile of circulating cytokines, including prototypical Th1, interferon- $\gamma$ (IFN- $\gamma$ ) (15) and Th-2 cytokines (IL-4, IL-13, IL-10) $(16,17)$, as well as IL-6 and TNF- $\alpha$, IL-7 and GM-CSF (18). In lung, cytokine production is disrupted by ethanol, exacerbating respiratory distress syndrome with upregulated expression of the Th3 and primarily anti-inflammatory cytokine, transforming growth factor- $\beta$ (TGF- $\beta$ ) $(19,20)$. In general, AUD patients, and in particular those with MDD, seem to exhibit higher levels of proinflammatory cy tokines, IL-6, IL-7, TNF- $\alpha$, GM-CSF, and IFN- $\gamma$, than controls $(18,21)$. One recent study has shown that high-risk drinkers showed significantly higher mean values of the proinflammatory cytokine IL-6 than abstainers (22). TNF- $\alpha$ and IL- 6 serum levels have been considered as possible neurobiological markers of alcohol consumption in AUD patients (23).

It has been clarified that chronic ethanol intake stimulates the immune system though activation of the NLRP3/ASC inflammasome. In turn, this leads to activation of caspase-1 and increase of IL-1 $\beta$ in the cerebellum. Accordingly, selective blockade of the IL-1/IL-1R signaling suppresses both the activation of the inflammasome and of neuroinflammation that are provoked by alcohol (24). In addition, the altered immune response evoked from alcohol abuse may in turn be implicated in the generation and maintenance of the mechanisms of alcohol addiction and regulation of drinking. For example, the activation of Toll-like receptor (TLR) 3 increases voluntary alcohol intake in C57BL/6J male (25). The potential clinical relevance of the interaction between the effects of AUD on the immune system and the possible role of this latter in regulating drinking has received further in vivo validation with the observation that TLR3-TIR-domain-containing adapter-inducing interferon- $\beta$ (TRIF) pathway are activated during chronic ethanol consumption. The IKKe/TBK1 inhibitor Amlexanox down-regulates TRIF-dependent pathway in the brain and reduces ethanol consumption, suggesting that the TRIF-dependent pathway is implicated in regulation of drinking (26). Hence, the NFKB pathway that lies downstream the activation of TLR signaling may also play a non canonical non-immune role in mediating complex behaviors, including processes of learning and memory, stress responses, anhedonia and drug reward (27). Along this line of research, in a translational study entailing both mice and humans, Coleman and coworkers generated strong proof of evidence in humans that HMGB1 and IL- $1 \beta$ heterocomplexes formed in vivo may contribute to the pathology of alcoholism. The Authors found that HMGB1/IL-1 $\beta$ complexes are increased in post-mortem human alcoholic hippocampus (28). These data have led to the hypothesis of alcohol-induced neuroimmune gene induction (29).

Taken as a whole, all these data strongly support the hypothesis that AUD can be characterized by generalized immune system dysfunction, caused by chronic alcohol exposure and supports the concept that alcoholism may represent a systemic proinflammatory condition (30). These data have attracted attention on the possible interaction between immunoinflammatory events that are induced by AUD and the possible role of immunoinflammatory events in the initiation and maintenance of AUD.

Along this line of research, a recent study has shown that the dual inhibitor of phosphodiesterase- 4 and -10 and of the pleiotropic cytokine macrophage migration inhibitory factor (MIF), Ibudilast (IBUD), exerts beneficial effects both in animal models of alcohol dependence (31) and in humans with AUD (32). On the basis of these data, we have presently studied eventual differences in transcriptomic levels of MIF and its homologue DDT along with their receptors CD74, CXCR2, CXCR4 and CXCR7 in the brain and peripheral blood of patients with AUD. The transcriptomic analysis of the present study demonstrates a trend to increased expression of MIF, DDT and CD74 in AUD patients. However, when expression levels of MIF and DDT were merged the expression of this superfamily was significantly higher than in healthy controls. These data indicate that dysregulation of MIF family members may occur during AUD and that specific tailored treatments with antagonists of MIF superfamily for AUD is worthy of further investigation. 


\section{Materials and methods}

\section{Microarray datasets selection and analysis}

Analysis of prefrontal cortex transcriptomic data. In order to investigate the expression levels of MIF and related genes in alcohol-related disorder, we interrogated the publicly-available whole-genome transcriptomic microarray datasets, GSE123114, GSE53808 and GSE49376, obtained from the Gene Expression Omnibus (GEO; https://www.ncbi.nlm. nih.gov/geo/) database. GSE123114 included whole genome transcriptomic data from an animal model of chronic alcohol self-administration (33). Briefly, for the generation of this dataset, mice were given free access to three bottles containing 5 and $10 \%$ of ethanol and water for 16 weeks. On the last two weeks, both ethanol solutions were adulterated with the aversive substance, quinine. Following this procedure, mice were classified into two groups, based on the ethanol intake and preference: 'Light Drinkers' (preference for water at all experimental stages); and 'Inflexible Drinkers' (preference for ethanol at all experimental stages, even after addition of quinine to the ethanol solution). At the end of the experimental period, total RNA was extracted from the Prefrontal cortex and the Affymetrix Mouse Genome 430 2.0 Array was used for the generation of the dataset. Details on the GSE123114 dataset can be obtained from the relative publication (33).

GSE53808 was used for the analysis of gene expression patterns in the prefrontal cortex of three alcoholics without hepatic encephalopathy and three neurologically normal controls (34). Brain tissues were obtained from the New South Wales Tissue Research Centre (NSW TRC), part-funded by the National Institute on Alcohol Abuse and Alcoholism (R24AA012725) (35). The Affymetrix Human Genome U219 Array was used for the generation of the dataset. For the analysis, technical replicates were averaged. Detailed description of the GSE53808 can be obtained from (35).

The GSE49376 dataset included whole-genome transcriptomic levels of the prefrontal cortex from 46 European Australians, obtained from the NSW TRC. Among the 46 subjects, 23 (16 males and 7 females) had alcohol use disorders, divided in alcohol-dependence $(n=9)$ and alcohol abuse $(n=14)$. The male and female samples did not significantly differ in age, postmortem intervals, brain $\mathrm{pH}$ and alcohol daily use. All subjects were not affected by illicit drug abuse and dependence or major psychotic disorders. The clinical information of all the patients are available in (36).

Analysis of blood transcriptomic data. GSE59206 included whole-genome blood transcriptomic data from two groups of subjects ( $\mathrm{n}=11 /$ group): Heavy drinkers (HD, defined as regular alcohol use over the past year of at least 8 standard drinks/week for women and at least 15 standard drinks/week for men), and moderate drinkers (MD, defined as up to 7 standard drinks/week for women and 14 standard drinks/week for men), as defined by the NIAAA. All subjects were between 21-50 years-old, had no history of any drug dependence (including alcohol dependence), and did not meet criteria for Axis I DSM-IV psychiatric diagnoses. Exclusion criteria included dependence on psychoactive substances; current or past use of opiates; current use of psychoactive drugs; psychotic disorders; neurological, cardiovascular, endocrine, renal, liver, thyroid diseases; subjects on medications for any medical condition; oral contraceptives; and pregnant and lactating women. Blood was collected at baseline and $1 \mathrm{~h}$ following exposure to an alcohol-related cue. Alcohol-related cue scripts were based on individual situations that included alcohol-related stimuli and resulted in subsequent alcohol use (e.g. buying alcohol and being at a bar, watching others drink alcohol). Microarray analysis was performed on blood cells using the Illumina HumanHT-12 V4.0 expression beadchip (37).

A summary of all the microarray datasets used in this study is presented in Table I.

Statistical analysis. Expression data are presented as mean \pm standard deviation (SD). The limma (Linear Models for Microarray Analysis) R package was used for identifying differentially expressed genes. An adjusted P-value $<0.05$ and a $\mid \log$ (fold change) $\mid>1$ was considered as threshold of significance.

Fisher's Inverse $\chi^{2}$ test was used as integrative analysis for MIF and DDT modulation in blood. This methods computes a combined statistic from the P-values obtained from the individual genes, as follows:

$$
\mathrm{S}=-2 \log \sum_{\mathrm{i}=0}^{\mathrm{n}}(\mathrm{pi})
$$

which follows a $\chi^{2}$ distribution with $2 \mathrm{n}$ degrees of freedom under the null hypothesis. Graphs were constructed using the GraphPad Prism 8 software.

\section{Results}

Analysis of brain MIF, DDT and receptors in murine and human alcohol abuse. Analysis of the transcriptomic levels of MIF, DDT and CD74 and the coreceptors, CXCR2, CXCR4 and CXCR7, in a murine model of alcohol dependence (GSE123114), revealed no significant modulation in Inflexible Drinkers as compared to Light Drinkers (Fig. 1A). In a similar manner, as determined in the GSE53808 dataset, in the prefrontal cortex, no significant differences between healthy control subjects and alcoholics were observed for MIF, DDT and their receptors (Fig. 1B). Similarly, as shown on Fig. 1C, alcohol abuse and dependence was not associated to significant alterations in the RNA levels of MIF, DDT and CD74 in the prefrontal cortex, as compared to healthy subjects (GSE49376). The expression levels of the coreceptors could not be evaluated in the latter dataset, as they were below the threshold of detection.

Analysis of blood MIF, DDT and receptors in heavy drinkers. The GSE59206 dataset was interrogated to evaluate the levels of MIF, DDT and their receptors/coreceptors in subjects at risk for alcohol dependence. As shown in Fig. 2A and B, heavy drinkers showed a moderate trend of increase in the levels of MIF and DDT, both at baseline and upon exposure to alcohol-related cues. Interestingly, alcohol-related cues did not, in the short term $(1 \mathrm{~h})$, determined a modulation in the levels of these two genes. Although the statistical significance was not reached, and in consideration of the overlapping effects of MIF and DDT (38), an inverse Fisher's chi square test was performed on unadjusted P-values to evaluate whether the combined effect of MIF and DDT modulation could be statistically significant. 
Table I. Characteristics of the microarray datasets analyzed in the study.

\begin{tabular}{|c|c|c|c|c|c|}
\hline Author, year & GEO identifier & Sample type & Samples & Platform & (Refs.) \\
\hline $\begin{array}{l}\text { da Silva e Silva et al, } \\
2016\end{array}$ & GSE123114 & Prefrontal cortex & $\begin{array}{l}3 \text { inflexible drinker mice } \\
\text { and } 3 \text { light drinker mice }\end{array}$ & $\begin{array}{l}\text { Affymetrix Mouse Genome } \\
4302.0 \text { Array }\end{array}$ & (33) \\
\hline Sutherland et al, 2014 & GSE53808 & $\begin{array}{l}\text { Prefrontal cortex } \\
\text { (frozen) }\end{array}$ & $\begin{array}{l}3 \text { alcoholics without } \\
\text { hepatic encephalopathy } \\
\text { and } 3 \text { neurologically } \\
\text { normal controls }\end{array}$ & $\begin{array}{l}\text { Affymetrix Human Genome } \\
\text { U219 Array }\end{array}$ & (34) \\
\hline Xu et al, 2014 & GSE49376 & $\begin{array}{l}\text { Prefrontal cortex } \\
\text { (frozen) }\end{array}$ & $\begin{array}{l}\text { Prefrontal cortex from } \\
46 \text { subjects, } 23 \text { ( } 16 \text { males } \\
\text { and } 7 \text { females) with alcohol } \\
\text { use disorders, divided in } \\
\text { alcohol-dependence }(n=9) \\
\text { and alcohol abuse }(n=14) \\
\text { and } 23 \text { controls }\end{array}$ & $\begin{array}{l}\text { Illumina HumanHT-12 V4.0 } \\
\text { expression beadchip }\end{array}$ & (36) \\
\hline Beech et al, 2014 & GSE59206 & Peripheral blood & $\begin{array}{l}11 \text { heavy drinkers and } 11 \\
\text { light drinkers }\end{array}$ & $\begin{array}{l}\text { Illumina HumanHT-12 V4.0 } \\
\text { expression beadchip }\end{array}$ & (37) \\
\hline
\end{tabular}
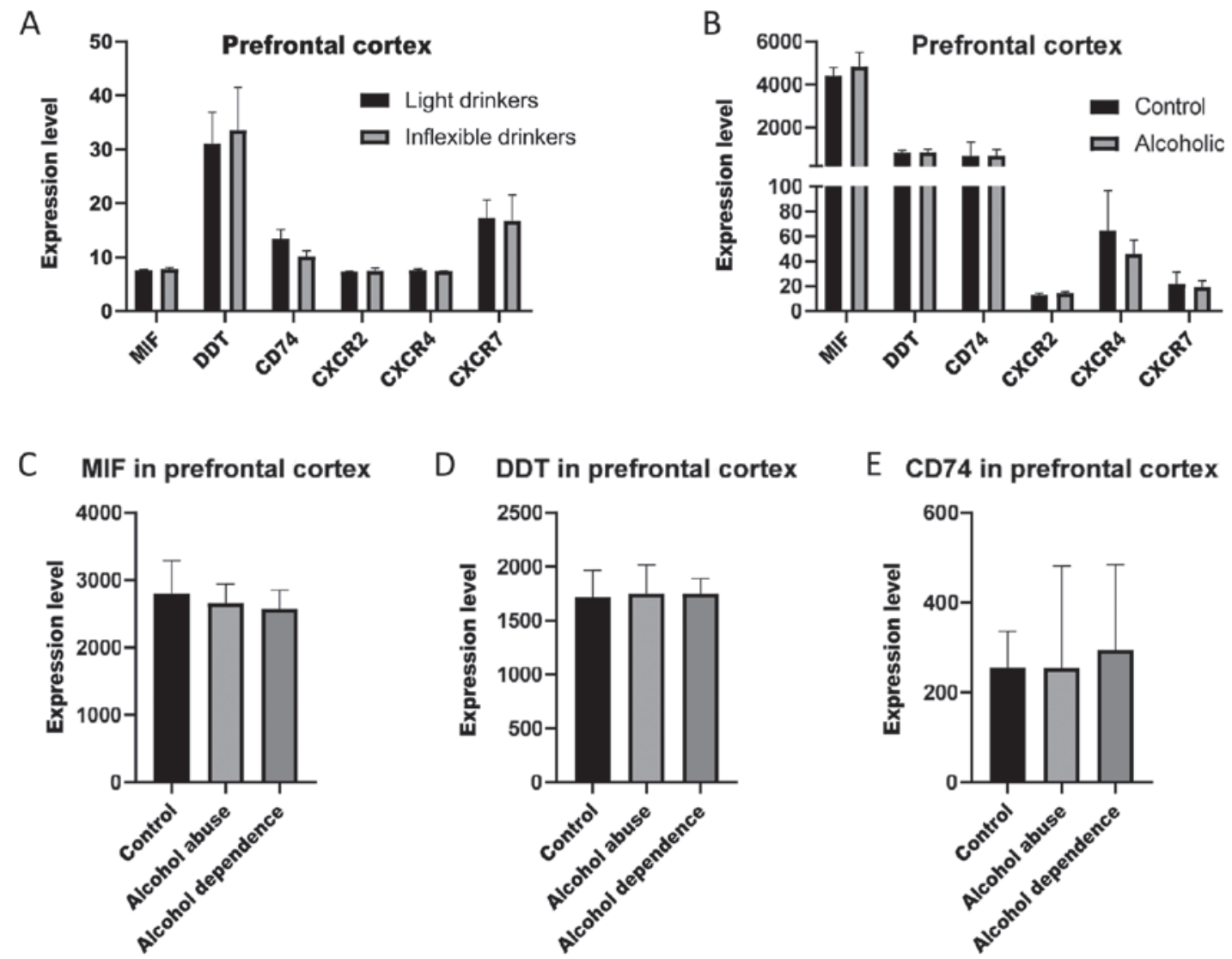

Figure 1. Expression of MIF superfamily and its receptors in the prefrontal cortex. (A) Transcriptomic data of murine prefrontal cortex tissue classified as 'light drinkers' and 'inflexible drinkers' based on ethanol intake and preference, as obtained from the GSE123114 dataset. (B) Analysis of gene expression patterns in the prefrontal cortex tissue of three alcoholics without hepatic encephalopathy and three neurologically normal controls, as obtained from the GSE53808 dataset. Expression levels of (C) MIF, (D) DD and (E) CD74 in prefrontal cortex tissue from 23 patients with alcohol use disorders. Patients were divided into alcohol-dependence $(n=9)$ and alcohol abuse $(n=14)$ groups, with 23 control subjects, as obtained from the GSE49376 dataset. MIF, migration inhibitory factor.

As expected, a combined p value of 0.042 was obtained at baseline and 0.016 at $1 \mathrm{~h}$ post-exposure (Fig. 2C and D). No significant modulation was observed for the receptor CD74 and the coreceptors, CXCR2, CXCR4 and CXCR7 (Fig. 2E-H). 

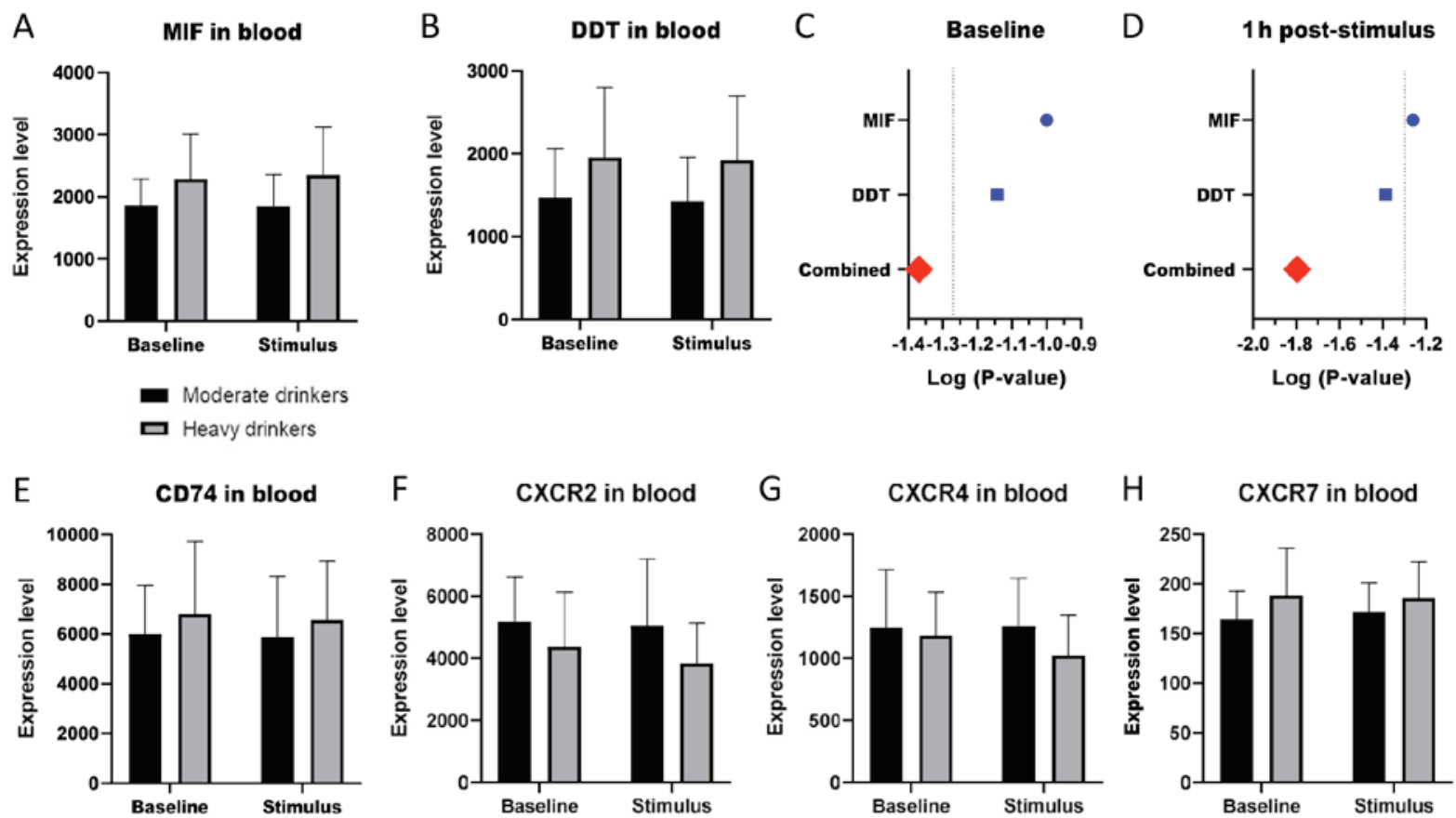

Figure 2. Expression of the MIF superfamily and its receptors in the blood cells of heavy drinkers. Transcriptomic levels of (A) MIF and (B) DDT in the blood cells of 11 heavy drinkers as compared with 11 moderate drinkers. Levels were assessed at the baseline and at $1 \mathrm{~h}$ post-exposure to an alcohol-related cue. Forrest plot showing combined P-values obtained by an Inverse Fisher's Chi Square test of MIF and DDT (C) at the baseline levels and at (D) $1 \mathrm{~h}$ post-exposure to an alcohol-related cue. Transcriptomic levels of (E) CD74, (F) CXCR2, (G) CXCR4 and (H) CXCR7 in the blood cells of 11 heavy drinkers compared with 11 moderate drinkers. Levels were assessed at the baseline and at $1 \mathrm{~h}$ post-exposure to an alcohol-related cue. Data were obtained from the GSE59206 microarray dataset. MIF, migration inhibitory factor; DDT, D-dopachrome tautomerase; CXC-R, C-X-C chemokine receptor.

\section{Discussion}

Increasing experimental and clinical evidence indicate that immunoinflammatory events are induced by alcohol exposure. It has been shown that microglia, the innate immune cells of the brain, and neurons respond to alcohol through receptors and molecules of the innate immune system, including, but not limited to, proinflammatory cytokines and their receptors $(39,40)$. The pathogenic relevance of immunoinflammatory events to the pathogenesis and maintenance of AUD has gained translational relevance with the demonstration of the beneficial effect obtained with minocycline in rodent models of AUD. However, clinical studies with this drug in human patients failed to confirm these preclinical findings (41). Nonetheless, the multiple data supporting a role for neuroinflammation in the pathogenesis of AUD have warranted further studies aimed at identifying other cells, molecules and receptors of the immune system that may play a role in the pathogenesis of the disorder and may eventually represent suitable therapeutic target.

MIF is a protein that has been discovered at the end of the 1960 s, and acquired its name from its ability to inhibit the migration of macrophages. The receptor of MIF is composed of the cell surface receptor CD74, that is associated with CD44, and of additional 3 non-cognate ligand for $\mathrm{C}-\mathrm{X}-\mathrm{C}$ chemokine receptor type 2 (CXCR2), type 4 (CXCR4) and type 7 (CXCR7) (38). A second member of the MIF family has also been identified in 2011, named D-dopachrome tautomerase (D-DT), that exhibits multiple synergisms with MIF [reviewed in (38)].

MIF, and more recently DDT, have been implicated in the pathogenesis of several pathologies, including immunoinflammatory and autoimmune diseases $(38,42-44)$ and cancer (45-47). As anticipated above, that MIF may represent an important pathogenic molecule in the pathogenesis of AUD emerges from recent studies indicating that the dual inhibitor of phosphodiesterase-4 and -10 and MIF, named IBUD, has beneficial effects of AUD both in rodent models and human patients $(31,32)$. In particular, this trial consisted of a randomized, crossover, double-blind, placebo-controlled laboratory study of IBUD in non treatment-seeking individuals that had suffered with mild-to-severe AUD, within the last month. The trial evaluated the safety, tolerability, and initial human laboratory efficacy of IBUD (50 $\mathrm{mg}$ b.i.d.) on primary measures of subjective response to alcohol, as well as secondary measures of cue- and stress-induced changes in craving and mood. IBUD was well tolerated but it failed to significantly influence primary measures. However, it significantly improved mood on the secondary measures of stress exposure and reduced tonic levels of craving. Furthermore, exploratory analyses indicated that IBUD preferentially influenced the stimulant and mood altering effects of alcohol as compared to placebo in those patients exhibiting the most severe depressive symptoms (39).

However, since IBUD is a dual inhibitor of PDE 4-10 and MIF, it is difficult to understand whether the effects in AUD is due to the combined synergistic pharmacological properties of IBUD or whether either of them accounts for by the beneficial effects of IBUD. It is therefore important to better identify which of the two pharmacological properties may be eventually relevant for the efficacy of drug in this condition. In addition, since it has recently been demonstrated that the MIF superfamily is composed from at least 2 different 
homologues, such as MIF and DDT, that most often, but not always, act synergistically. It is also important to shed light on the possible combined action that MIF, DDT and their receptors, play in development and maintenance of AUD. For this reason, we carried out a transcriptomic study that evaluated the expression of MIF, DDT and their receptors in the brains of AUD patients and in peripheral blood cells of people at risk of developing AUD, under basal conditions and upon exposure to alcohol-related cues. In addition, we also evaluated the transcriptomic analysis of MIF, DDT and their receptors in the brains of a mouse model of alcoholism. This analysis was carried out by DNA microarray analysis that represents a useful in silico toll for the better understanding of pathogenic pathways and the possible prediction of new diagnostic therapeutic approaches in several clinical settings, including immunoinflammatory and autoimmune diseases and fibrotic diseases $(45,46,48-59)$. Our analysis first demonstrated that no differences in the expression of MIF, DDT and their receptors can be found in brains of both rodent models of AUD and patients with this condition. However, when peripheral blood of heavy drinkers was studied, it was possible to observe a consistent, though not statistically significant, trend, of increased expression of MIF and DDT in the blood of heavy drinkers as compared to healthy controls, both at baseline and upon exposure to alcohol-related cues. Interestingly, knowing the overlapping effects of MIF and DDT in several biological responses (38) we performed the inverse Fisher's $\chi^{2}$ test on unadjusted $\mathrm{p}$ values and found out that the combined effect of MIF and DDT modulation was statistically significant as compared to controls. Along with the trend of augmented expression of the CD74 receptors in the peripheral blood of heavy drinkers as compared to controls these data seem to indicate that a hyperactivation of the MIF superfamily may also occur in AUD patients. This finding is of particular relevance for its possible translation, as it has recently been demonstrated that dual specific antagonists of MIF and DDT, such as 4-iodo-6-phenylpyrimidine (4-IPP), have been developed (60). Hence, this new class of drugs could be worthy being studied in rodent models of AUD in a head to head comparison with single MIF inhibitors and eventually IBUD. This is of further particular relevance as MIF has been implicated in the pathogenesis of other forms of dependence and addiction that could benefit from specific dual inhibitors of MIF and DDT. Although our data are the first to demonstrate that a moderate upregulated transcriptomic expression of the MIF-DDT axis occurs in the blood of people at risk of developing AUD, other studies are necessary to fully understand the exact role of MIF and DDT in development and maintenance of AUD. In particular, it will be important to prove whether selected groups of AUD patients can be identified on the basis of their MIF-DDT secretory capacity and whether those with highest expression profile are eventually those that may respond better to tailored anti MIF-DDT therapies. Studies of genetic polymorphism of MIF and DDT in AUD patients may be warranted. It will also be important to determine on larger number of patients whether in a manner similar to progressive multiple sclerosis, MIF and DDT are more abundantly produced in males than in females (61), as this may further facilitate identification of individual potentially responders to anti-MIF-DDT. In addition, it will be relevant to measure circulating levels of MIF and DDT in AUD patients at different time points during their development and maintenance of addiction, as our transcriptomic analysis do not take into account the amount of MIF and DDT that can be secreted from resident cells, such as epithelial and endothelial cells that are not represented in peripheral blood samples. Nonetheless, in spite of the needs of these further studies, our present analysis provides in vivo evidence for the upregulated synthesis of MIF and DDT in AUD and highlights the possibility that these molecules may offer novel important diagnostic and therapeutic opportunities in prevention and treatment of AUD along with psychotherapeutic approaches.

\section{Acknowledgements}

Not applicable.

\section{Funding}

The present study was supported by the current research funds 2019 of IRCCS ‘Centro Neurolesi ‘Bonino Pulejo’ (Messina-Italy).

\section{Availability of data and materials}

The datasets analyzed in the current study are available from the Gene Expression Omnibus website (GEO; https://www.ncbi. nlm.nih.gov/geo/) with accession nos. GSE123114, GSE53808, GSE49376 and GSE59206.

\section{Authors' contributions}

MCP, EM, FN and EC conceived and designed the present study. $\mathrm{KM}, \mathrm{PF}, \mathrm{RDM}$ and $\mathrm{LF}$ analyzed the data and prepared the figures. MCP, KM, MSB and EC interpreted the data and wrote the manuscript. EM, RDM and FN critically revised the manuscript. All authors read and approved the final version of the manuscript.

\section{Ethics approval and consent to participate}

Not applicable.

\section{Patient consent for publication}

Not applicable.

\section{Competing interests}

The authors declare that they have no competing interests.

\section{References}

1. Wang X, Zhang K, Yang F, Ren Z, Xu M, Frank JA, Ke ZJ and Luo J: Minocycline protects developing brain against ethanol-induced damage. Neuropharmacology 129: 84-99, 2018.

2. Debell F, Fear NT, Head M, Batt-Rawden S, Greenberg N, Wessely S and Goodwin L: A systematic review of the comorbidity between PTSD and alcohol misuse. Soc Psychiatry Psychiatr Epidemiol 49: 1401-1425, 2014.

3. Pietrzak RH, Goldstein RB, Southwick SM and Grant BF: Prevalence and Axis I comorbidity of full and partial posttraumatic stress disorder in the United States: Results from Wave 2 of the national epidemiologic survey on alcohol and related conditions. J Anxiety Disord 25: 456-465, 2011. 
4. Neupane SP, Bramness JG and Lien L: Comorbid post-traumatic stress disorder in alcohol use disorder: Relationships to demography, drinking and neuroimmune profile. BMC Psychiatry 17: $312,2017$.

5. Franck J and Jayaram-Lindström N: Pharmacotherapy for alcohol dependence: Status of current treatments. Curr Opin Neurobiol 23: 692-699, 2013.

6. Soyka M and Müller CA: Pharmacotherapy of alcoholism-an update on approved and off-label medications. Expert Opin Pharmacother 18: 1187-1199, 2017.

7. Hillemacher T and Frieling H: Pharmacotherapeutic options for co-morbid depression and alcohol dependence. Expert Opin Pharmacother 20: 547-569, 2019.

8. Garbutt JC: The state of pharmacotherapy for the treatment of alcohol dependence. J Subst Abuse Treat 36: S15-S25, 2009.

9. Szabo G, Mandrekar P, Petrasek J and Catalano D: The unfolding web of innate immune dysregulation in alcoholic liver injury. Alcohol Clin Exp Res 35: 782-786, 2011.

10. Qin L, He J, Hanes RN, Pluzarev O, Hong JS and Crews FT: Increased systemic and brain cytokine production and neuroinflammation by endotoxin following ethanol treatment. J Neuroinflammation 5: 10, 2008.

11. Kalejaiye O, Getachew B, Ferguson CL, Taylor RE and Tizabi Y: Alcohol-induced increases in inflammatory cytokines are attenuated by nicotine in region-selective manner in male rats. J Drug Alcohol Res 6: pii: 236036, 2017.

12. McClain C, Barve S, Deaciuc I, Kugelmas M and Hill D: Cytokines in alcoholic liver disease. Semin Liver Dis 19: 205-219, 1999

13. McClain CJ and Cohen DA: Increased tumor necrosis factor production by monocytes in alcoholic hepatitis. Hepatology 9: 349-351, 1989

14. Ahluwalia B, Wesley B, Adeyiga O, Smith DM, Da-Silva A and Rajguru S: Alcohol modulates cytokine secretion and synthesis in human fetus: an in vivo and in vitro study. Alcohol 21: 207-213, 2000.

15. Nicoletti F, Zaccone P, Di Marco R, Lunetta M, Magro G, Grasso S, Meroni P and Garotta G: Prevention of spontaneous autoimmune diabetes in diabetes-prone BB rats by prophylactic treatment with antirat interferon-gamma antibody. Endocrinology 138: 281-288, 1997.

16. Nicoletti F, Mancuso G, Cusumano V, Di Marco R, Zaccone P Bendtzen K and Teti G: Prevention of endotoxin-induced lethality in neonatal mice by interleukin-13. Eur J Immunol 27: $1580-1583,1997$.

17. Marchant A, Bruyns C, Vandenabeele P, Ducarme M, Gérard C, Delvaux A, De Groote D, Abramowicz D, Velu T and Goldman M: Interleukin-10 controls interferon-gamma and tumor necrosis factor production during experimental endotoxemia. Eur J Immunol 24: 1167-1171, 1994.

18. González-Reimers E, Santolaria-Fernández F, Medina-García JA, González-Pérez JM, de la Vega-Prieto MJ, Medina-Vega L, Martín-González C and Durán-Castellón MC: TH-1 and TH-2 cytokines in stable chronic alcoholics. Alcohol Alcohol 47: 390-396, 2012.

19. Nicoletti F, Di Marco R, Patti F, Reggio E, Nicoletti A, Zaccone P, Stivala F, Meroni PL and Reggio A: Blood levels of transforming growth factor-beta 1 (TGF-beta1) are elevated in both relapsing remitting and chronic progressive multiple sclerosis (MS) patients and are further augmented by treatment with interferon-beta $1 \mathrm{~b}$ (IFN-beta1b). Clin Exp Immunol 113: 96-99, 1998.

20. Crews FT, Bechara R, Brown LA, Guidot DM, Mandrekar P, Oak S, Qin L, Szabo G, Wheeler M and Zou J: Cytokines and alcohol. Alcohol Clin Exp Res 30: 720-730, 2006.

21. Nikou T, Ioannidis A, Zoga M, Tzavellas E, Paparrigopoulos T, Magana M, Pliatsika P, Nikolaou C and Chatzipanagiotou S: Alteration in the concentrations of Interleukin-7 (IL-7), Interleukin-10 (IL-10) and Granulocyte Colony Stimulating Factor (G-CSF) in alcohol-dependent individuals without liver disease, during detoxification therapy. Drug Alcohol Depend 163 : 77-83, 2016.

22. Archer M, Kampman O, Bloigu A, Bloigu R, Luoto K, Kultti J, Hämäläinen $M$, Moilanen E, Leinonen E and Niemelä $O$ : Assessment of alcohol consumption in depression follow-up using self-reports and blood measures including inflammatory biomarkers. Alcohol Alcohol 54: 243-250, 2019.

23. Heberlein A, Käser M, Lichtinghagen R, Rhein M, Lenz B, Kornhuber J, Bleich S and Hillemacher T: TNF- $\alpha$ and IL-6 serum levels: Neurobiological markers of alcohol consumption in alcohol-dependent patients? Alcohol 48: 671-676, 2014
24. Lippai D, Bala S, Petrasek J, Csak T, Levin I, Kurt-Jones EA and Szabo G: Alcohol-induced IL-1 $\beta$ in the brain is mediated by NLRP3/ASC inflammasome activation that amplifies neuroinflammation. J Leukoc Biol 94: 171-182, 2013.

25. Warden AS, Azzam M, DaCosta A, Mason S, Blednov YA, Messing RO, Mayfield RD and Harris RA: Toll-like receptor 3 activation increases voluntary alcohol intake in C57BL/6J male mice. Brain Behav Immun 77: 55-65, 2019.

26. McCarthy GM, Warden AS, Bridges CR, Blednov YA and Harris RA: Chronic ethanol consumption: Role of TLR3/TRIF-dependent signaling. Addict Biol 23: 889-903, 2018.

27. Nennig SE and Schank JR: The role of NFkB in drug addiction: Beyond inflammation. Alcohol Alcohol 52: 172-179, 2017.

28. Coleman LG Jr, Zou J, Qin L and Crews FT: HMGB1/IL-1 $\beta$ complexes regulate neuroimmune responses in alcoholism. Brain Behav Immun 72: 61-77, 2018.

29. Crews FT and Vetreno RP: Mechanisms of neuroimmune gene induction in alcoholism. Psychopharmacology (Berl) 233: 1543-1557, 2016.

30. González-Reimers E, Santolaria-Fernández F, MartínGonzález MC, Fernández-Rodríguez CM and Quintero-Platt G: Alcoholism: A systemic proinflammatory condition. World J Gastroenterol 20: 14660-14671, 2014.

31. Bell RL, Lopez MF, Cui C, Egli M, Johnson KW, Franklin KM and Becker HC: Ibudilast reduces alcohol drinking in multiple animal models of alcohol dependence. Addict Biol 20: 38-42, 2015.

32. Ray LA, Bujarski S, Shoptaw S, Roche DJ, Heinzerling K and Miotto K: Development of the neuroimmune modulator ibudilast for the treatment of alcoholism: Arandomized, placebo-controlled, human laboratory trial. Neuropsychopharmacology 42: 1776-1788, 2017.

33. da Silva e Silva DA, Frozino Ribeiro A, Damasceno S, Rocha CS, Berenguer de Matos AH, Boerngen-Lacerda R, Correia D and Brunialti Godard AL: Inflexible ethanol intake: A putative link with the Lrrk2 pathway. Behav Brain Res 313: 30-37, 2016.

34. Sutherland GT, Sheedy D, Sheahan PJ, Kaplan W and Kril JJ: Comorbidities, confounders, and the white matter transcriptome in chronic alcoholism. Alcohol Clin Exp Res 38: 994-1001, 2014.

35. Sheedy D, Garrick T, Dedova I, Hunt C, Miller R, Sundqvist N and Harper C: An Australian brain bank: A critical investment with a high return! Cell Tissue Bank 9: 205-216, 2008.

36. Xu H, Wang F, Liu Y, Yu Y, Gelernter J and Zhang H: Sex-biased methylome and transcriptome in human prefrontal cortex. Hum Mol Genet 23: 1260-1270, 2014.

37. Beech RD, Leffert JJ, Lin A, Hong KA, Hansen J, Umlauf S, Mane S, Zhao H and Sinha R: Stress-related alcohol consumption in heavy drinkers correlates with expression of miR-10a, miR-21, and components of the TAR-RNA-binding protein-associated complex. Alcohol Clin Exp Res 38: 2743-2753, 2014.

38. Günther S, Fagone P, Jalce G, Atanasov AG, Guignabert C and Nicoletti F: Role of MIF and D-DT in immune-inflammatory, autoimmune, and chronic respiratory diseases: From pathogenic factors to therapeutic targets. Drug Discov Today 24: 428-439, 2019.

39. Crews FT, Lawrimore CJ, Walter TJ and Coleman LG: The role of neuroimmune signaling in alcoholism. Neuropharmacology 122: 56-73, 2017

40. Alfonso-Loeches S, Pascual-Lucas M, Blanco AM, SanchezVera I and Guerri C: Pivotal role of TLR4 receptors in alcohol-induced neuroinflammation and brain damage. J Neurosci 30: 8285-8295, 2010.

41. Petrakis IL, Ralevski E, Gueorguieva R, Sloan ME, Devine L, Yoon G, Arias AJ and Sofuoglu M: Targeting neuroinflammation with minocycline in heavy drinkers. Psychopharmacology (Berl) 236: 3013-3021, 2019.

42. Nicoletti F, Mazzon E, Fagone P, Mangano K, Mammana S, Cavalli E, Basile MS, Bramanti P, Scalabrino G, Lange A and Curtin F: Prevention of clinical and histological signs of MOG-induced experimental allergic encephalomyelitis by prolonged treatment with recombinant human EGF. J Neuroimmunol 332: 224-232, 2019.

43. Fagone P, Mazzon E, Cavalli E, Bramanti A, Petralia MC, Mangano K, Al-Abed Y, Bramati P and Nicoletti F: Contribution of the macrophage migration inhibitory factor superfamily of cytokines in the pathogenesis of preclinical and human multiple sclerosis: In silico and in vivo evidences. J Neuroimmunol 322: 46-56, 2018. 
44. Cavalli E, Mazzon E, Basile MS, Mangano K, Di Marco R, Bramanti P, Nicoletti F, Fagone P and Petralia MC: Upregulated expression of macrophage migration inhibitory factor, its analogue D-dopachrome tautomerase, and the CD44 receptor in peripheral CD4 $\mathrm{T}$ cells from clinically isolated syndrome patients with rapid conversion to clinical defined multiple sclerosis. Medicina (Kaunas) 55: pii: E667, 2019.

45. Lombardo SD, Presti M, Mangano K, Petralia MC, Basile MS, Libra M, Candido S, Fagone P, Mazzon E, Nicoletti F and Bramanti A: Prediction of PD-L1 expression in neuroblastoma via computational modeling. Brain Sci 9: pii: E221, 2019.

46. Presti M, Mazzon E, Basile M, Petralia MC, Bramanti A, Colletti G, Bramanti P, Nicoletti F and Fagone P: Overexpression of macrophage migration inhibitory factor and functionally-related genes, D-DT, CD74, CD44, CXCR2 and CXCR4, in glioblastoma. Oncol Lett 16: 2881-2886, 2018.

47. Mangano K, Mazzon E, Basile MS, Di Marco R, Bramanti P, Mammana S, Petralia MC,Fagone P and Nicoletti F: Pathogenic role for macrophage migration inhibitory factor in glioblastoma and its targeting with specific inhibitors as novel tailored therapeutic approach. Oncotarget 9: 17951-17970, 2018.

48. Fagone P, Mangano K, Mammana S, Pesce A, Pesce A, Caltabiano R, Giorlandino A, Portale TR, Cavalli E, Lombardo GA, et al: Identification of novel targets for the diagnosis and treatment of liver fibrosis. Int J Mol Med 36: 747-752, 2015.

49. Lombardo SD, Mazzon E, Basile MS, Campo G, Corsico F, Presti M, Bramanti P, Mangano K, Petralia MC, Nicoletti F and Fagone P: Modulation of tetraspanin 32 (TSPAN32) expression in T cell-mediated immune responses and in multiple sclerosis. Int J Mol Sci 20: pii: E4323, 2019.

50. Candido S, Lupo G, Pennisi M, Basile MS, Anfuso CD Petralia MC, Gattuso G, Vivarelli S, Spandidos DA, Libra $\mathrm{M}$ and Falzone L: The analysis of miRNA expression profiling datasets reveals inverse microRNA patterns in glioblastoma and Alzheimer's disease. Oncol Rep 42: 911-922, 2019.

51. Petralia MC, Mazzon E, Fagone P, Falzone L, Bramanti P, Nicoletti F and Basile MS: Retrospective follow-up analysis of the transcriptomic patterns of cytokines, cytokine receptors and chemokines at preconception and during pregnancy, in women with post-partum depression. Exp Ther Med 18: 2055-2062, 2019.

52. Mangano K, Lanteri R, Basile MS, Bellavia N, Latino R, Messina D, Fagone P, Colletti G, Nania R, Caltabiano R, et al: Effects of GIT-27NO, a NO-donating compound, on hepatic ischemia/reperfusion injury. Int J Immunopathol Pharmacol 33: $2058738419862736,2019$.
53. Lombardo SD, Mazzon E, Basile MS, Cavalli E, Bramanti P, Nania R, Fagone P, Nicoletti F and Petralia MC: Upregulation of IL-1 receptor antagonist in a mouse model of migraine. Brain Sci 9: pii: E172, 2019.

54. Mammana S, Bramanti P, Mazzon E, Cavalli E, Basile MS, Fagone P, Petralia MC, McCubrey JA, Nicoletti F and Mangano K: Preclinical evaluation of the PI3K/Akt/mTOR pathway in animal models of multiple sclerosis. Oncotarget 9: 8263-8277, 2018.

55. Mangano K, Cavalli E, Mammana S, Basile MS, Caltabiano R, Pesce A, Puleo S, Atanasov AG, Magro G, Nicoletti F and Fagone P: Involvement of the Nrf2/HO-1/CO axis and therapeutic intervention with the CO-releasing molecule CORM-A1, in a murine model of autoimmune hepatitis. J Cell Physiol 233: 4156-4165, 2018.

56. Petralia MC, Mazzon E, Fagone P, Russo A, Longo A, Avitabile T, Nicoletti F, Reibaldi M and Basile MS: Characterization of the pathophysiological role of CD47 in uveal melanoma. Molecules 24: pii: E2450, 2019.

57. Basile MS, Fagone P, Mangano K, Mammana S, Magro G, Salvatorelli L, Li Destri G, La Greca G, Nicoletti F, Puleo S and Pesce A: KCNMA1 expression is downregulated in colorectal cancer via epigenetic mechanisms. Cancers (Basel) 11: pii: E245, 2019.

58. Basile MS, Mazzon E, Russo A, Mammana S, Longo A, Bonfiglio V, Fallico M, Caltabiano R, Fagone P, Nicoletti F, et al: Differential modulation and prognostic values of immune-escape genes in uveal melanoma. PLoS One 14: e0210276, 2019.

59. Fagone P, Caltabiano R, Russo A, Lupo G, Anfuso CD, Basile MS, Longo A, Nicoletti F, De Pasquale R, Libra M and Reibaldi M: Identification of novel chemotherapeutic strategies for metastatic uveal melanoma. Sci Rep 7: 44564, 2017.

60. Rajasekaran D, Zierow S, Syed M, Bucala R, Bhandari V and Lolis EJ: Targeting distinct tautomerase sites of D-DT and MIF with a single molecule for inhibition of neutrophil lung recruitment. FASEB J 28: 4961-4971, 2014.

61. Benedek G, Meza-Romero R, Jordan K, Zhang Y, Nguyen H, Kent G, Li J, Siu E, Frazer J, Piecychna M, et al: MIF and D-DT are potential disease severity modifiers in male MS subjects. Proc Natl Acad Sci USA 114: E8421-E8429, 2017.

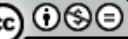

This work is licensed under a Creative Commons Attribution-NonCommercial-NoDerivatives 4.0 International (CC BY-NC-ND 4.0) License. 